\title{
APD volumetric app: android app for the quantification of reagents
}

\author{
Ian Hartono Budianto ${ }^{1}$ and Samuel Ken-En Gan ${ }^{1,2,3^{*}}$
}

\begin{abstract}
Quantifying reagents is a necessity in laboratory experiment planning but to do so with reasonable accuracy is a challenge when using unmarked containers. While there are volumetric measuring cylinders, the demand of sterility and purity in many experiments prevent their use in a feasible fashion. As a result, experienced estimation is usually relied upon, but this is fairly subjective, and may lead to large margins of error. To ease this process, APD Volumetric App, a stand-alone native Android application enables the smartphone to calculate the volume of the substance in a container through image analysis. Using pixel ratios, a much more accurate estimation of the remaining liquid can be made. This allows the rapid quantification of the contents without the need of volumetric cylinders, especially when dealing with very minute quantities. With this app, more accurate estimations to keep track of reagents for logistics, leisure, and operations, can be performed in an easily streamlined and convenient manner.
\end{abstract}

\section{Introduction}

Once a device for leisure and productivity in everyday life, the smartphone has been leveraged on increasingly for clinical uses (see review Gan SKE et al., 2016) and in biomedical research (see review Gan SKE \& Poon JK, 2016). On this, a significant number of apps have been developed for daily operations in scientific settings. Some examples from our previous work function to replace the lab computer (e.g. DNAapp in Nguyen et al., 2014), some for more convenient data collection (e.g. PsychVey in Nguyen et al., 2015), and some to replace equipment and automated existing processes (e.g. GelApp in Sim et al., 2015; APD Colony Counter App in Wong et al., 2016). In the same vein of automating certain processes, there is room for the development of apps for processes that are not yet routine, but can be implemented as a process to smoothen planning and logistics.

In the daily course of many experiments in scientific research, there are requirements to determine the remaining volume of reagents when planning for experiments for logistic purposes. Similarly, with increasing safety audits on the usage of controlled substances/chemicals (e.g. List I and List II chemicals in USA Drug Enforcement Administration website), there is often a requirement to quickly determine the remaining volume of substances for records keeping or even for audits. While measuring cylinders are accurate, their use would not be suitable for use on hazardous chemicals (e.g. powder), on very minute amounts e.g. the amount of enzymes in microliters $(\mu \mathrm{l})$ in microfuge tubes, or in cases where sterility, temperature sensitivity, and purity of the solution must be maintained (e.g. biological samples such as competent cells, see Chan et al., 2013).

For the above mentioned purposes, an image based quantification of substances would be beneficial for quick estimation, and thus we made the APD Volumetric App for this purpose. Leveraging on image processing methods and the smartphone camera to analyze user-defined cropped images, the user can conveniently and quickly estimate the remaining contents in a container. By simply

\footnotetext{
*Correspondence: biip53apd@gmail.com; samuelg@bii.a-star.edu.sg

${ }^{1}$ Bioinformatics Institute, Agency for Science, Technology, and Research

(A*STAR), Singapore 138671, Singapore

${ }^{2}$ p53 Laboratory, Agency for Science, Technology, and Research (A*STAR),

Singapore 138648, Singapore

Full list of author information is available at the end of the article
} 

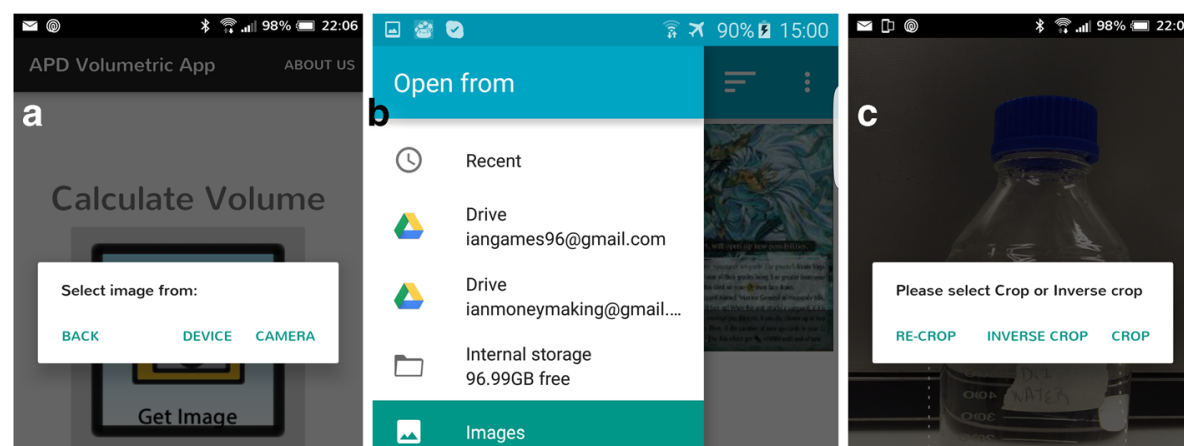

\section{$\Delta \quad$ Images}

(c) Downloads

6. Gallery

e Photos

D Dropbox

OneDrive

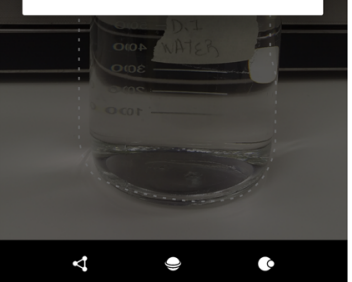

Fig. 1 Starting APD Volumetric App. a Choice of loading the picture from the device or to capture a new image directly from the camera. b Loading of the desired image from the phone, and also from Cloud storage apps. c Loaded image for processing

taking a picture with the application or by loading a previous image, users can estimate the remaining volume after from the auto-calculation of content space from the known maximum volume of the container.

Since the smartphone is portable, quantities of controlled or hazardous chemicals can be conveniently determined on-the-go without the need for direct exposure to biological and chemical hazards. In the planning of experiments that utilize microliter quantities such as molecular cloning, it is now possible to estimate with better accuracy, that half drop of enzyme in the tubes.
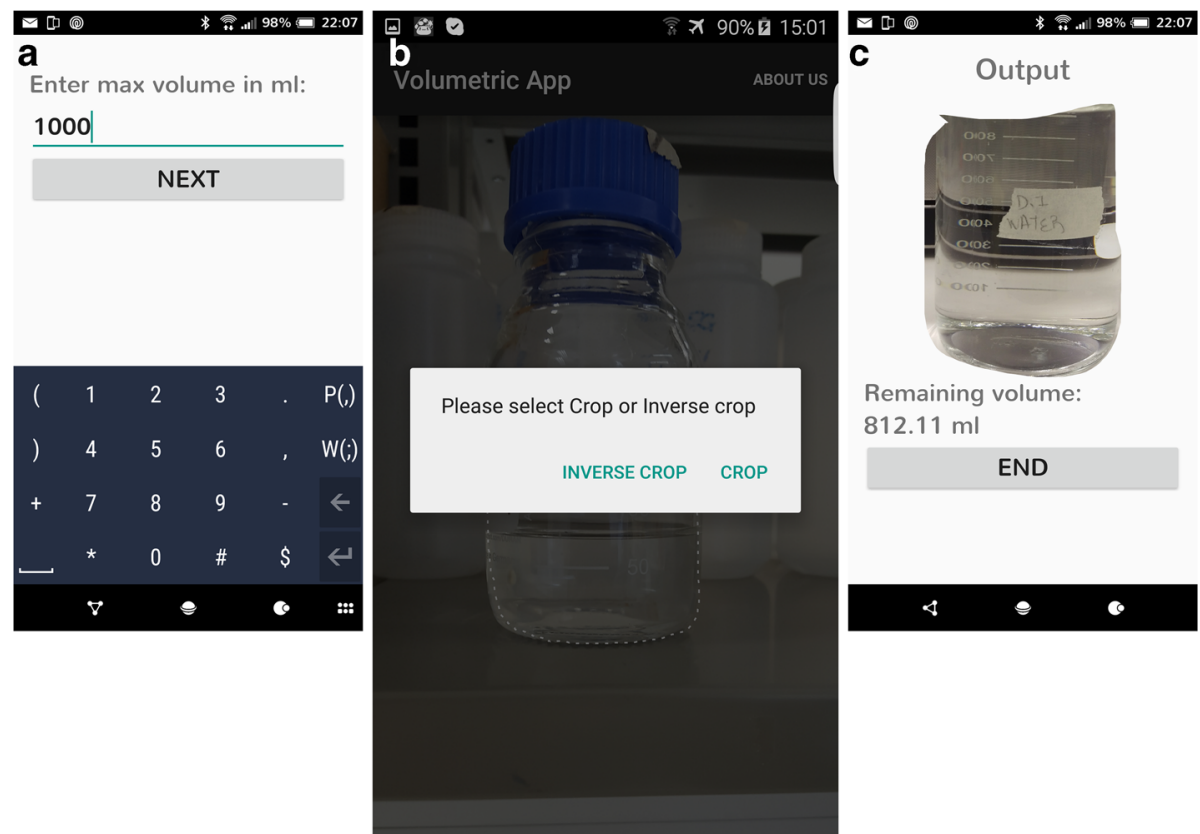

Fig. 2 Determining the Volume. a Entering the maximum volume of the cropped image. $\mathbf{b}$ Cropping of the contents. $\mathbf{c}$ Results of the calculation of the remaining contents 


\section{Development and methodology}

The APD Volumetric App for Android was developed using version 4.2 of the Eclipse Integrated Development Environment, Juno.

\section{Calculation of volume}

The app allows users to use direct camera capture or loading of existing images for analysis (Fig. 1a, b). Users are then to trace the outline of the container, either with their finger or for higher accuracy, with associated smartphone pens. For more accurate results, users are advised to take a close up of the container with the meniscus as a straight line. The cropped areas are marked by a dotted line.

Should there be inaccurate cropping, the cropping process can be restarted with the "RE-CROP" button. In the unlikely event when it is easier to crop the areas outside the container (e.g. when the object exceeds the image size), the "INVERSE CROP" function can be used. Otherwise, when the user is satisfied that the dotted line traces the container accurately, they can select the area with the "CROP" function (Fig. 1c).

The user is next prompted to enter the known maximum volume of the cropped container (Fig. 2).

Following which, the user is prompted to crop the area of the remaining contents in the same manner. Based on the formulae as shown in Fig. 3, the remaining volume of the contents would be calculated.

The formula used to calculate volume is as follows:

The formula uses the total number of pixels forming the container and the entered known volume of the container to determine the amount of volume represented by a single pixel. By determining the volume represented by a single pixel, the application will be able to calculate the amount of liquid present in the container by multiplying it by the number of pixels the liquid occupies.

As the volume calculations are determined by the pixels, the accuracy of the final calculated value is highly dependent on the outlining process than on the angle of the image. In this sense, to fully tap on the app, it is advisable to use a smartphone pen (generic ones with the soft round ball at the end, or specialized pens that are present in Microsoft Surface Pro series, Samsung Tablets, and Samsung Galaxy Note series) to outline the images. Should there be some odd shape or angles, the user may choose to make appropriate compensations in the outlining process.$$
\frac{\text { Maximum volume of container }}{\text { Total number of pixels of container }} \times \begin{gathered}
\text { Total number of pixels } \\
\text { of liquid present }
\end{gathered}
$$

Fig. 3 Formula used to calculate volume of liquid present

\section{Application and limitations}

To our knowledge, APD Volumetric App is the only app in the Google Play Store that works to quantify the content of round-bottomed or cylindrical objects such as beakers, test tubes, and microfuge tubes as these are the typical shapes in biomedical research labs. Particularly designed to quantify the minute amounts in

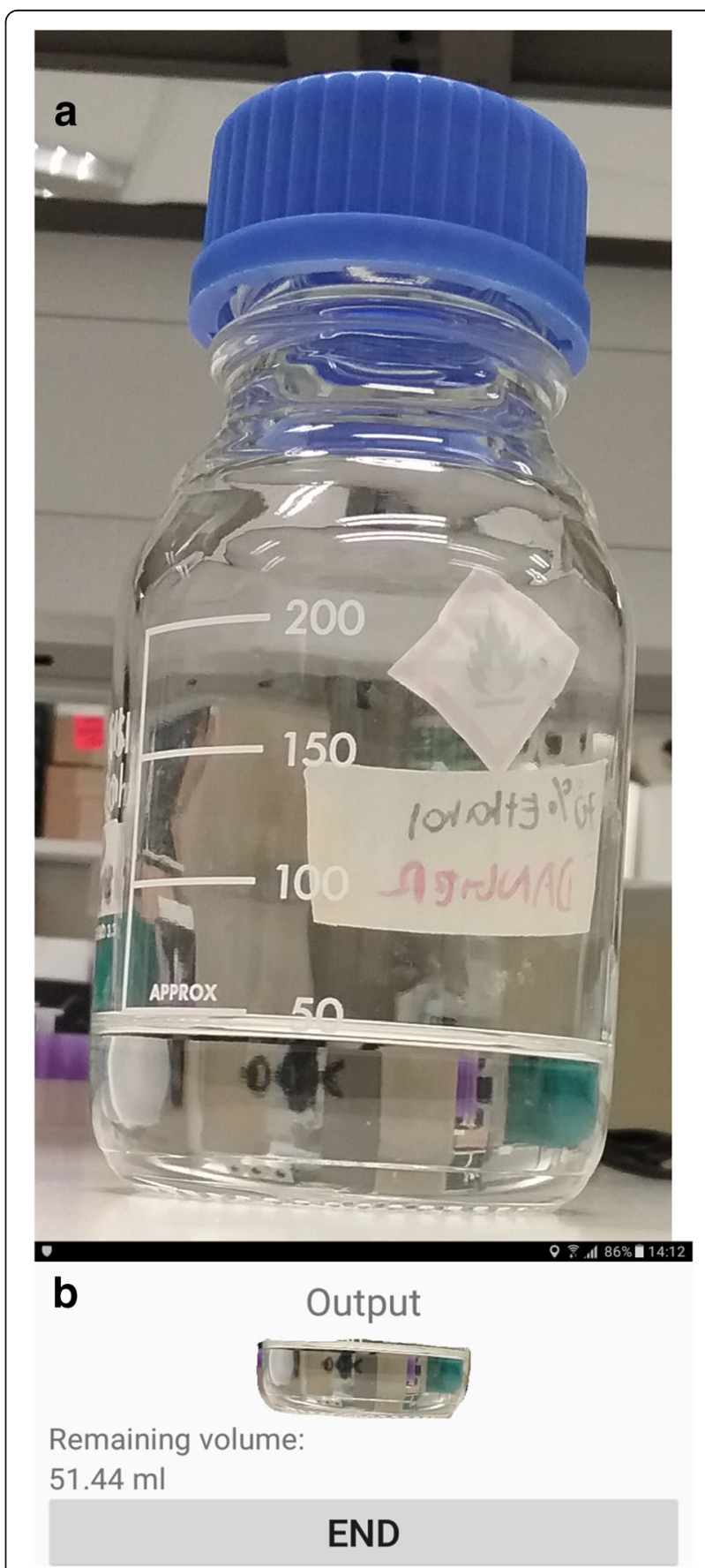

Fig. 4 Accuracy check of the APD Volumetric App with a laboratory beaker. a Image of $50 \mathrm{mls}$ in a lab beaker. $\mathbf{b}$ Quantification of $50 \mathrm{mls}$ in the lab beaker as $51.44 \mathrm{ml}$ using APD Volumetric App 
microfuge tubes, the app also has applications in common daily processes (e.g. cooking) and in quick logistical checks (liquid stocktaking). These applications can include gauging the amount of liquid supplied in containers, verifying the accuracy of markings of containers, estimating large amounts or very minute volumes, and in estimating the amount of a beverage in a glass e.g. wine in a glass or bottle.

In our tests of $50 \mathrm{ml}(\mathrm{ml})$ of liquid in a $500 \mathrm{ml}$ beaker, we were able to get the result of $51.44 \mathrm{ml}$ estimation (Fig. 4), demonstrating that under accurate cropping conditions, fairly accurate estimates of the volume could be obtained. Due to the image processing nature, the container has to be transparent or at least, translucent for the gauging of the level of the contents. Care must be taken that liquid meniscus should be as straight as possible at camera lens level to reduce parallax errors that will give rise to inaccurate estimates.

As the app is targeted for a safe and convenient quantification in an indirect manner, the app is not designed to be of a replacement of volumetric cylinders given the natural limitations of image processing and dependence on user cropping.

\section{Future work}

To cater to more varieties of bottles, including square bottomed containers (e.g. fish tanks) calculation methods would be incorporated. Auto-cropping features are also being developed.

\section{Conclusion}

APD Volumetric App is a standalone Android application that brings convenience and efficiency to volume measurement. The app does not require internet connectivity to support its features, and allows users to measure the volume of liquid present in a container without the need of physical volumetric measurements.

\section{Acknowledgements}

We thank Mr. Cornelius Koshy for proofreading.

\section{Funding}

Not applicable

\section{Availability of data and materials}

The APD Volumetric App application is developed for Android and is freely available on the Google Play Store. More details on the app can be found at www.facebook.com/APDLab; www.bii.a-star.edu.sg/research/trd/apd.php.

The APD Volumetric App user guide is available at http://tinyurl.com/ APDVolumeApp, linked in the 'About Us' page of the app. A video tutorial is also available on the Google Play Store.

\section{Authors' contributions}

IHB made the app and drafted the document. SKE oversaw and directed the design and making of the apps. Both authors have read and approved of the manuscript.

\section{Competing interests}

The authors declare that they have no competing interests.

\section{Author details}

${ }^{1}$ Bioinformatics Institute, Agency for Science, Technology, and Research (A*STAR), Singapore 138671, Singapore. ${ }^{2}$ p53 Laboratory, Agency for Science, Technology, and Research (A*STAR), Singapore 138648, Singapore. ${ }^{3}$ APD

SKEG Pte Ltd, Singapore 439444, Singapore.

Received: 19 January 2017 Accepted: 11 October 2017

Published online: 03 November 2017

\section{References}

Chan WT, Verma CS, Lane DP, Gan SK. A comparison and optimization of methods and factors affecting the transformation of Escherichia Coli. Biosci Rep. 2013;33(6):art:e00086. doi:10.1042/BSR20130098.

Gan SKE, Poon JK. The world of biomedical apps: their uses, limitations, and potential. Scientific Phone Apps and Mobile Devices. 2016;2:6. doi:10.1186/ s41070-016-0009-2.

Gan SKE, Koshy C, Nguyen PV, Haw YX. An overview of clinically and healthcare related apps in Google and apple app stores: connecting patients, drugs, and clinicians. Scientific phone apps and mobile devices, vol. 3; 2016.

Nguyen PV, Lim JPH, Budianto IH, Gan SKE (2015). PsychVey: Research Survey App. Scientific Phone Apps and Mobile Devices, Vol 1(3), doi:10.1186/s41070015-0002-1.

Nguyen PV, Verma CS, Gan SKE. DNAApp: a mobile application for sequencing data analysis. Bioinformatics, vol. 2014. p. 2014. doi:10.1093/bioinformatics/btu525.

Sim JZ, Nguyen PV, Lee HK, Gan SKE. GelApp: mobile gel electrophoresis analyzer. Nature Methods Application Notes. 2015; doi:10.1038/an9643.

Wong CF, Yeo JY. Gan SKE (2016) APD Colony counter app: using watershed algorithm for improved colony counting. Nature Methods Application Notes. Aug 2016; doi:10.1038/ an9774.

\section{Submit your manuscript to a SpringerOpen ${ }^{\mathcal{O}}$ journal and benefit from:}

- Convenient online submission

- Rigorous peer review

- Open access: articles freely available online

- High visibility within the field

- Retaining the copyright to your article

Submit your next manuscript at springeropen.com 